\title{
Asymptotic results for silent elimination
}

\author{
Guy Louchard ${ }^{1}$ and Helmut Prodinger ${ }^{2} \|^{\dagger}$ \\ ${ }^{1}$ Université Libre de Bruxelles, Dép. d'Informatique, CP 212, B-1050 Bruxelles, Belgium. Iouchardeulb. ac.be \\ ${ }^{2}$ Stellenbosch University, Department of Mathematics, 7602 Stellenbosch, South Africa. hproding@ sun.ac. za
}

received May 24, 2009, revised Jan. 3, 2010, accepted Jan. 18, 2010.

Following the model of Bondesson, Nilsson, and Wikstrand, we consider randomly filled urns, where the probability of falling into urn $i$ is the geometric probability $(1-q) q^{i-1}$. Assuming $n$ independent random entries, and a fixed parameter $k$, the interest is in the following parameters: Let $T$ be the smallest index, such that urn $T$ is non-empty, but the following $k$ are empty, then: $X_{T}=$ number of balls in urn $T, S_{T}=$ number of balls in urns with index larger than $T$, and finally $T$ itself.

We analyse the recursions (that appeared earlier) precisely, and derive results about the joint distribution of a related urn model.

Keywords: Silent elimination, gaps, urns, Poisson generating function, Mellin transform, recursion, joint distribution

\section{Introduction}

Dedicated to Philippe Flajolet

In (1) the following situation was discussed, which resembles the following urn model:

Assume that there are urns labelled $1,2, \ldots$, and $n$ balls thrown into the urns at random, independently, according to the geometric distribution: the probability that a ball goes into urn $j$ is $p q^{j-1}$ with $p+q=1$.

Now let $k \geq 1$ be a fixed integer, and we consider the smallest index $T$ such that

- $\operatorname{urn} T$ is non-empty,

- urns $T+1, T+2, \ldots, T+k$ are empty.

Then the idea is that $T$ (or the balls in the urn indexed by $T$ ) can be considered as a pseudo winner, since it is not very likely that after $k$ empty urns there are still elements in some urns with higher index.

The instance $k=\infty$ corresponds to the highest index of a non-empty urn. The index $T$ in this case is very classical, we just cite $(15,8)$. The distribution of the number of balls in this urn has also been subject of several papers; we just cite three: $(2,3 ; 9)$.

The instance $k=1$ refers to the first gap; $T$ is in this case one less than the index of the first unoccupied urn. This parameter has a rich history and is the driving force behind probabilistic counting (4); our paper (12) contains also some pointers to more recent papers.

\footnotetext{
${ }^{\dagger}$ This material is based upon work supported by the National Research Foundation under grant number 2053748 1365-8050 @ 2010 Discrete Mathematics and Theoretical Computer Science (DMTCS), Nancy, France
} 
The authors of (1) consider 3 parameters (random variables), which depend on $n$ : In the simplified urn model, which is easier to understand, they correspond to

- $X_{T}$, the number of balls in urn $T$,

- $S_{T}$, the number of balls in urns with index $>T+k$,

- $T$.

For the respective expected values, recursions were derived, but no asymptotic evaluations were given. We fill in these gaps, and show that $\mathbb{E}_{n}\left(X_{T}\right) \sim$ const $+\delta(\log n), \mathbb{E}_{n}\left(S_{T}\right) \sim$ const $+\delta(\log n)$ and $\mathbb{E}_{n}(T) \sim \log n+$ const $+\delta(\log n)$, where $Q=q^{-1}, L=\ln Q, \log \equiv \log _{Q}$ and $\delta(x)$ is a periodic function of period 1 with mean zero and small amplitude. We use the notation in a generic sense: in different instances it might mean a different function; the Fourier coefficients could be computed in principle, but we refrain from doing it.

This is only part of a more ambitious project: We want to use our machinery described in (11) and compute all moments. However, that is not easy, and we did not succeed thus far.

We start with the recursions provided in (1) (exactly as given there, not the urn model) and then use the following multistep procedure that is described in several textbooks, notably in (14):

- The recursion is translated into a functional equation for the exponential generating function $A(z)$.

- This functional equation is translated in terms of the Poisson generating function $B(z)=e^{-z} A(z)$, with the motive that $a_{n}=n !\left[z^{n}\right] A(z) \sim B(n)$.

- To find $B(z)$ for a large parameter $z$, we use the Mellin transform, $B^{*}(s)$ and the inversion formula

$$
B(z)=\frac{1}{2 \pi i} \int_{-\frac{1}{2}-i \infty}^{-\frac{1}{2}+i \infty} B^{*}(s) z^{-s} d s .
$$

- This integral will be evaluated via residues. The line of integration will be shifted to the right, and the residues (with a minus sign) will be collected.

\section{Recursions for the expected values}

Theorem 1 (Theorem 2 in (1)) The expected values $\mathbb{E}_{n}\left(X_{T}\right)$ are recursively given by

$$
\mathbb{E}_{n}\left(X_{T}\right)=\frac{1-q^{n k}}{1-q^{n}} n p\left(p+q^{k-1}\right)^{n-1}+\frac{1-q^{n k}}{1-q^{n}} \sum_{j=1}^{n}\left(\begin{array}{c}
n \\
j
\end{array}\right) p^{j} q^{n-j} \mathbb{E}_{n-j}\left(X_{T}\right)
$$

and $\mathbb{E}_{0}\left(X_{T}\right)=0$.

Theorem 2 (Theorem 3 in (1)) The expected values $\mathbb{E}_{n}\left(S_{T}\right)$ are recursively given by

$$
\mathbb{E}_{n}\left(S_{T}\right)=n q^{n k}+\frac{1-q^{n k}}{1-q^{n}} \sum_{j=1}^{n}\left(\begin{array}{c}
n \\
j
\end{array}\right) p^{j} q^{n-j} \mathbb{E}_{n-j}\left(S_{T}\right)
$$

and $\mathbb{E}_{0}\left(S_{T}\right)=0$. 
Theorem 3 (Theorem 4 in (1)) The expected values $\mathbb{E}_{n}(T)$ are recursively given by

$$
\mathbb{E}_{n}(T)=\frac{1-q^{n k}}{1-q^{n}}-k q^{n k}+\frac{1-q^{n k}}{1-q^{n}} \sum_{j=1}^{n}\left(\begin{array}{c}
n \\
j
\end{array}\right) p^{j} q^{n-j} \mathbb{E}_{n-j}(T)
$$

and $\mathbb{E}_{0}(T)=0$

\section{Asymptotic study of $\mathbb{E}_{n}\left(S_{T}\right)$}

Let us write $a_{n}=\mathbb{E}_{n}\left(S_{T}\right)$, then the recursion is equivalent to

$$
a_{n}\left(1-q^{n(k+1)}\right)=n q^{n k}\left(1-q^{n}\right)+\left(1-q^{n k}\right) \sum_{j=0}^{n}\left(\begin{array}{c}
n \\
j
\end{array}\right) p^{j} q^{n-j} a_{n-j} .
$$

Now we set

$$
A(z):=\sum_{n \geq 0} a_{n} \frac{z^{n}}{n !}
$$

and translate:

$$
A(z)-A\left(z q^{k+1}\right)=z q^{k} e^{z q^{k}}-z q^{k+1} e^{z q^{k+1}}+e^{p z} A(z q)-e^{p q^{k} z} A\left(z q^{k+1}\right) .
$$

Now we introduce the Poisson generating function $B(z)=e^{-z} A(z)$ :

$$
B(z)-e^{-z} A\left(z q^{k+1}\right)=z q^{k} e^{-z\left(1-q^{k}\right)}-z q^{k+1} e^{-z\left(1-q^{k+1}\right)}+B(z q)-e^{-z\left(1-p q^{k}\right)} A\left(z q^{k+1}\right) .
$$

This is of the form

$$
B(z)-B(z q)=R(z),
$$

where $R(z)$ is a "harmless" function. The idea of our procedure is, as described in the book (14), that $B(n) \sim a_{n}$, and the behaviour of $B(z)$ for large $z$ will be determined by the Mellin transform. We find

$$
B^{*}(s)=\frac{R^{*}(s)}{1-q^{-s}}
$$

The fundamental strip is $\langle-1,0\rangle$. Furthermore,

$$
\begin{aligned}
R^{*}(s)= & \int_{0}^{\infty}\left(z q^{k} e^{-z\left(1-q^{k}\right)}-z q^{k+1} e^{-z\left(1-q^{k+1}\right)}+e^{-z} A\left(z q^{k+1}\right)-e^{-z\left(1-p q^{k}\right)} A\left(z q^{k+1}\right)\right) z^{s-1} d z \\
= & \frac{q^{k}}{\left(1-q^{k}\right)^{s+1}} \Gamma(s+1)-\frac{q^{k+1}}{\left(1-q^{k+1}\right)^{s+1}} \Gamma(s+1) \\
& \quad+\int_{0}^{\infty}\left(e^{-z}-e^{-z\left(1-p q^{k}\right)}\right) \sum_{j \geq 0} a_{j} \frac{z^{j} q^{j(k+1)}}{j !} z^{s-1} d z
\end{aligned}
$$




$$
\begin{aligned}
=\frac{q^{k}}{\left(1-q^{k}\right)^{s+1}} \Gamma(s+ & 1)-\frac{q^{k+1}}{\left(1-q^{k+1}\right)^{s+1}} \Gamma(s+1) \\
& +\sum_{j \geq 0} a_{j} \frac{q^{j(k+1)}}{j !} \Gamma(s+j)\left(1-\frac{1}{\left(1-p q^{k}\right)^{s+j}}\right) .
\end{aligned}
$$

Therefore

$$
-\operatorname{res}_{s=0}\left\{\frac{R^{*}(s) z^{-s}}{1-q^{-s}}\right\}=\frac{1}{L} \frac{q^{k}}{1-q^{k}}-\frac{1}{L} \frac{q^{k+1}}{1-q^{k+1}}+\frac{1}{L} \sum_{j \geq 0} a_{j} \frac{q^{j(k+1)}}{j}\left(1-\frac{1}{\left(1-p q^{k}\right)^{j}}\right) .
$$

Theorem 4 Using the shorthand notation $a_{n}=\mathbb{E}_{n}\left(S_{T}\right)$, we have the asymptotic formula:

$$
\mathbb{E}_{n}\left(S_{T}\right) \sim \frac{1}{L} \frac{q^{k}}{1-q^{k}}-\frac{1}{L} \frac{q^{k+1}}{1-q^{k+1}}+\frac{1}{L} \sum_{j \geq 0} a_{j} \frac{q^{j(k+1)}}{j}-\frac{1}{L} \sum_{j \geq 0} a_{j} \frac{q^{j(k+1)}}{\left(1-p q^{k}\right)^{j} j}+\delta(\log n) .
$$

\section{Asymptotic study of $\mathbb{E}_{n}\left(X_{T}\right)$}

Let us write $a_{n}=\mathbb{E}_{n}\left(X_{T}\right)$, then the recursion is equivalent to

$$
a_{n}\left(1-q^{n(k+1)}\right)=\left(1-q^{n k}\right) n p\left(p+q^{k-1}\right)^{n-1}+\left(1-q^{n k}\right) \sum_{j=0}^{n}\left(\begin{array}{c}
n \\
j
\end{array}\right) p^{j} q^{n-j} a_{n-j} .
$$

It holds for $n \geq 0$. Now we set

$$
A(z):=\sum_{n \geq 0} a_{n} \frac{z^{n}}{n !}
$$

and translate:

$$
A(z)-A\left(z q^{k+1}\right)=p z e^{z\left(p+q^{k-1}\right)}-p q^{k} z e^{z q^{k}\left(p+q^{k-1}\right)}+e^{p z} A(z q)-e^{p q^{k} z} A\left(z q^{k+1}\right) .
$$

In terms of the Poisson transformed function it is

$B(z)-B(z q)=p z e^{-z\left(1-\left(p+q^{k-1}\right)\right)}-p q^{k} z e^{-z\left(1-q^{k}\left(p+q^{k-1}\right)\right)}+e^{-z} A\left(z q^{k+1}\right)-e^{-\left(1-p q^{k}\right) z} A\left(z q^{k+1}\right)$.

The Mellin transform of the righthand side is

$$
\frac{p \Gamma(s+1)}{\left(q-q^{k-1}\right)^{s+1}}-\frac{p q^{k} \Gamma(s+1)}{\left(1-p q^{k}-q^{2 k-1}\right)^{s+1}}+\sum_{j \geq 0} a_{j} \frac{q^{(k+1) j}}{j !} \Gamma(s+j)-\sum_{j \geq 0} a_{j} \frac{q^{(k+1) j}}{j !} \frac{\Gamma(s+j)}{\left(1-p q^{k}\right)^{s+j}},
$$

and thus

$$
\begin{aligned}
B^{*}(s)=\frac{1}{1-q^{-s}}\left(\frac{p \Gamma(s+1)}{\left(q-q^{k-1}\right)^{s+1}}-\right. & \frac{p q^{k} \Gamma(s+1)}{\left(1-p q^{k}-q^{2 k-1}\right)^{s+1}} \\
& \left.+\sum_{j \geq 0} a_{j} \frac{q^{(k+1) j}}{j !} \Gamma(s+j)-\sum_{j \geq 0} a_{j} \frac{q^{(k+1) j}}{j !} \frac{\Gamma(s+j)}{\left(1-p q^{k}\right)^{s+j}}\right) .
\end{aligned}
$$


¿From this, we find:

$$
-\operatorname{res}_{s=0}\left\{B^{*}(s) z^{-s}\right\}=\frac{1}{L}\left(\frac{p}{q-q^{k-1}}-\frac{p q^{k}}{1-p q^{k}-q^{2 k-1}}+\sum_{j \geq 0} a_{j} \frac{q^{(k+1) j}}{j}-\sum_{j \geq 0} a_{j} \frac{q^{(k+1) j}}{j} \frac{1}{\left(1-p q^{k}\right)^{j}}\right) .
$$

Theorem 5 Using the shorthand notation $a_{n}=\mathbb{E}_{n}\left(X_{T}\right)$, we have the asymptotic formula:

$$
\mathbb{E}_{n}\left(X_{T}\right) \sim \frac{1}{L}\left(\frac{p}{q-q^{k-1}}-\frac{p q^{k}}{1-p q^{k}-q^{2 k-1}}+\sum_{j \geq 0} a_{j} \frac{q^{(k+1) j}}{j}-\sum_{j \geq 0} a_{j} \frac{q^{(k+1) j}}{j} \frac{1}{\left(1-p q^{k}\right)^{j}}\right)+\delta(\log n) .
$$

\section{Asymptotic study of $\mathbb{E}_{n}(T)$}

Let us write $a_{n}=\mathbb{E}_{n}(T)$, then the recursion is equivalent to

$$
\begin{gathered}
a_{n}\left(1-q^{n(k+1)}\right)=1-(k+1) q^{n k}+k q^{n(k+1)}+\left(1-q^{n k}\right) \sum_{j=0}^{n}\left(\begin{array}{c}
n \\
j
\end{array}\right) p^{j} q^{n-j} a_{n-j} ; \\
A(z)-A\left(z q^{k+1}\right)=e^{z}-(k+1) e^{z q^{k}}+k e^{z q^{k+1}}+e^{p z} A(z q)-e^{p q^{k} z} A\left(z q^{k+1}\right) ; \\
B(z)-B(z q)=1-(k+1) e^{-z\left(1-q^{k}\right)}+k e^{-z\left(1-q^{k+1}\right)}+e^{-z} A\left(z q^{k+1}\right)-e^{-z\left(1-p q^{k}\right)} A\left(z q^{k+1}\right) .
\end{gathered}
$$

The Mellin transform of

$$
-(k+1) e^{-z\left(1-q^{k}\right)}+k e^{-z\left(1-q^{k+1}\right)}+e^{-z} A\left(z q^{k+1}\right)-e^{-z\left(1-p q^{k}\right)} A\left(z q^{k+1}\right)
$$

is

$$
-\frac{k+1}{\left(1-q^{k}\right)^{s}} \Gamma(s)+\frac{k}{\left(1-q^{k+1}\right)^{s}} \Gamma(s)+\sum_{j \geq 0} a_{j} \frac{q^{(k+1) j}}{j !} \Gamma(j+s)-\sum_{j \geq 0} a_{j} \frac{q^{(k+1) j}}{\left(1-p q^{k}\right)^{j+s} j !} \Gamma(j+s) .
$$

The fundamental strip is $\langle 0, \infty\rangle$.

This is also the transform of

$$
1-(k+1) e^{-z\left(1-q^{k}\right)}+k e^{-z\left(1-q^{k+1}\right)}+e^{-z} A(z q)-e^{-z\left(1-p q^{k}\right)} A\left(z q^{k+1}\right),
$$

but the fundamental strip is $\langle-1,0\rangle$.

Now, since we have terms $\Gamma(s)$, we expect a double pole, coming from the first two terms.

We need

$$
\begin{aligned}
& -\operatorname{res}_{s=0}\left\{\frac { z ^ { - s } } { 1 - q ^ { - s } } \left(-\frac{k+1}{\left(1-q^{k}\right)^{s}} \Gamma(s)+\frac{k}{\left(1-q^{k+1}\right)^{s}} \Gamma(s)\right.\right. \\
& \left.\left.\quad+\sum_{j \geq 0} a_{j} \frac{q^{(k+1) j}}{j !} \Gamma(j+s)-\sum_{j \geq 0} a_{j} \frac{q^{(k+1) j}}{\left(1-p q^{k}\right)^{j+s} j !} \Gamma(j+s)\right)\right\} \\
& =\operatorname{res}_{s=0}\left\{\frac{z^{-s}}{1-q^{-s}}\left(\frac{k+1}{\left(1-q^{k}\right)^{s}}-\frac{k}{\left(1-q^{k+1}\right)^{s}}\right) \Gamma(s)\right\}+\frac{1}{L} \sum_{j \geq 0} a_{j} \frac{q^{(k+1) j}}{j}-\frac{1}{L} \sum_{j \geq 0} a_{j} \frac{q^{(k+1) j}}{\left(1-p q^{k}\right)^{j} j} .
\end{aligned}
$$


The remaining residue is computed as follows:

$$
\begin{aligned}
& \operatorname{res}_{s=0}\left\{\frac{z^{-s}}{1-q^{-s}}\left(\frac{k+1}{\left(1-q^{k}\right)^{s}}-\frac{k}{\left(1-q^{k+1}\right)^{s}}\right) \Gamma(s)\right\} \\
& =\left[s^{-1}\right] \frac{1-s \ln z}{-L s\left(1+\frac{1}{2} L s\right)}\left(\frac{k+1}{1+s \ln \left(1-q^{k}\right)}-\frac{k}{1+s \ln \left(1-q^{k+1}\right)}\right)\left(\frac{1}{s}-\gamma\right) \\
& =\log z-\left[s^{1}\right] \frac{1-\frac{1}{2} L s}{L}\left(1-(k+1) s \ln \left(1-q^{k}\right)+k s \ln \left(1-q^{k+1}\right)\right)(1-s \gamma) \\
& =\log z+\frac{1}{2}+\frac{\gamma}{L}+(k+1) \log \left(1-q^{k}\right)-k \log \left(1-q^{k+1}\right) .
\end{aligned}
$$

Theorem 6 Using the shorthand notation $a_{n}=\mathbb{E}_{n}(T)$, we have the asymptotic formula:

$$
\begin{aligned}
\mathbb{E}_{n}(T) & \sim \log n+\frac{1}{2}+\frac{\gamma}{L}+(k+1) \log \left(1-q^{k}\right)-k \log \left(1-q^{k+1}\right) \\
& +\frac{1}{L} \sum_{j \geq 0} a_{j} \frac{q^{(k+1) j}}{j}-\frac{1}{L} \sum_{j \geq 0} a_{j} \frac{q^{(k+1) j}}{\left(1-p q^{k}\right)^{j} j}+\delta(\log n) .
\end{aligned}
$$

\section{The asymptotic joint distributions}

In this section, we concentrate on the joint distribution of the 3 parameters, $\mathbb{P}\left[T=\ell, X_{T}=i, S_{T}=j\right]$, which is quite involved, even in the simplified urn model, which we assume now. Next we consider $U:=$ number of gaps in a sequence of $n$ geometric RVs with parameter $p$ (gaps are defined below). Then we analyze $M L$ :=maximum gaps length. Finally we return to the joint distribution of $X_{T}$ and $S_{T}$ (independently of $T$ ). All asymptotics in this section are related to $n \rightarrow \infty$. We obtain explicit expressions and periodic contributions. Again $k$ is fixed here.

We summarize the notations that we use, although some have already appeared in earlier sections:

$$
\begin{aligned}
Q & :=1 / q, \\
L & :=\ln 1 / q=\ln Q, \\
n^{*} & :=n p / q, \\
\log & :=\log _{Q}, \\
\beta & :=q / p, \\
\chi_{l} & :=2 l \pi \mathbf{i} / L, \quad l \in \mathbb{Z} \backslash\{0\}
\end{aligned}
$$

Let us consider the model as a sequence of $n$ geometric iid RVs, with distribution $p q^{i-1}$. We have the following properties:

- We have asymptotic independence of urns, for all events related to $j=\mathcal{O}(\log m)$. This is proved, by Poissonization-DePoissonization, in (12), (13) and (7) (in this paper for $p=1 / 2$, but the proof is easily adapted). The error term is $\mathcal{O}\left(m^{-C}\right)$ where $C$ is a positive constant.

- We obtain asymptotic distibutions of the interesting RVs. The number of balls in each urn is now Poisson-distributed with parameter $n^{*} q^{j}$ in urn $j$. The asymptotic distibutions are related to Gumbel distributions functions or convergent series of such. The error term is $\mathcal{O}\left(\mathrm{m}^{-1}\right)$. 
- We have uniform integrability for the moments of our RVs. To show that the limiting moments are equivalent to the moments of the limiting distributions, we need a suitable rate of convergence. This is related to a uniform integrability condition (see Loève (10, Section 11.4)). For the kind of limiting distributions we consider here, the rate of convergence is analyzed in detail in (11) and (13). The error term is $\mathcal{O}\left(m^{-C}\right)$.

- Asymptotic expressions for the moments are obtained by Mellin transforms. The error term is $\mathcal{O}\left(m^{-C}\right)$.

We have here the asymptotic expression

\section{Theorem 6.1}

$$
\begin{aligned}
\mathbb{P}\left[T=\ell, X_{T}=i, S_{T}=j\right] & \sim e^{-n^{*} q^{\ell}} \frac{\left(n^{*} q^{\ell}\right)^{i}}{i !} e^{-n^{*} q^{\ell+1}\left(1-q^{k}\right) / p} e^{-n^{*} q^{\ell+k+1} / p} \frac{\left(n^{*} q^{\ell+k+1} / p\right)^{j}}{j !} \times \\
& {\left[\sum_{v=1}^{k-1} e^{-n^{*} q^{\ell-v}\left(1-q^{v}\right) / p} P\left[n^{*}, \ell-v-1\right]+P\left[n^{*}, \ell-1\right]\right], }
\end{aligned}
$$

where $P\left(n^{*}, u\right)$ is the probability that urn $u$ is non-empty and below either all urns are non-empty, or there are $r-1 \geq 0$ non-empty urns and below a gap of length $t<k$ and below no gaps of length $\geq k$. (In the last sum, the extra term is the one for index $v=0$.)

\section{Proof:}

We use the Poisson property. Urn $\ell$ is not empty (with $i$ balls) and above we have $k$ empty urns and above we have $j$ balls.

Below urn $\ell$ we have either $v<k$ empty urns and below one non-empty urn and below no gaps of length $\geq k$, or one non-empty urn and below no gaps of length $\geq k$.

$P\left(n^{*}, u\right)$ is the probability that urn $u$ is non-empty and below either all urns are non-empty, or there are $r-1 \geq 0$ non-empty urns and below a gap of length $t<k$ and below no gaps of length $\geq k$.

This means

$$
P\left[n^{*}, u\right]=\prod_{v=u}^{-\infty}\left(1-e^{-n^{*} q^{v}}\right)+\sum_{r=1}^{\infty} \prod_{v=u}^{u-r+1}\left(1-e^{-n^{*} q^{v}}\right) \sum_{t=1}^{k-1} e^{-n^{*} q^{u-r-t}\left(1-q^{t}\right) / p} P\left[n^{*}, u-r-t-1\right] .
$$

Set

$$
n^{*} q^{u}=\frac{n^{*}}{Q^{u}}=x
$$

and the recurrence becomes, setting $v-u=s$,

$$
P[x]=\prod_{s=0}^{\infty}\left(1-e^{x Q^{s}}\right)+\sum_{r=1}^{\infty} \prod_{s=0}^{r-1}\left(1-e^{-x Q^{s}}\right) \sum_{t=1}^{k-1} e^{-x Q^{r+t}\left(1-Q^{-t}\right) / p} P\left[x Q^{r+t+1}\right] .
$$

We couldn't solve recurrence (2) up to now. It does not seem possible to obtain a simpler form for the correlation between our three variables. 
If we sum on $\ell$ in (1) (i.e., we are interested in the joint distribution of $X_{T}$ and $S_{T}$ ), we are now in the field of gaps analysis, which has attracted some interest recently. A gap is a maximal sequence of contiguous empty urns, below the last non-empty urn: see Hitczenko and Knopfmacher (6), Goh and Hitczenko (5), Louchard and Prodinger (12) (in this paper, the weak gaps are analyzed, i.e., the number of empty urns below the last non-empty urn).

Let us first analyze $U:=$ number of gaps in a sequence of $n$ geometric RVs with parameter $p$. Set $p_{n}(u):=\mathbb{P}[U=u]$ and $F_{n}(u):=\sum_{i=0}^{u} p_{n}(i)$. We have

Theorem 6.2

$$
\begin{gathered}
p_{n}(u) \sim \sum_{\ell=1}^{\infty}\left[1-e^{\left.-n^{*} q^{\ell}\right]} e^{-n^{*} q^{\ell+1} / p} \prod_{v=1}^{\infty}\left[1-e^{\left.-n^{*} q^{\ell-v}\right]}\right.\right. \\
\sum_{r_{1}=1}^{\infty} \sum_{d_{1}=1}^{\infty} \frac{\exp \left[-n^{*} q^{\ell-r_{1}-d_{1}+1}\left(1-q^{d_{1}}\right) / p\right]}{\prod_{\ell_{1}=0}^{d_{1}-1}\left[1-e^{\left.-n^{*} q^{\ell-r_{1}-\ell_{1}}\right]}\right.} \\
\quad \sum_{r_{2}=r_{1}+d_{1}+1}^{\infty} \sum_{d_{2}=1}^{\infty} \frac{\exp \left[-n^{*} q^{\ell-r_{2}-d_{2}+1}\left(1-q^{d_{2}}\right) / p\right]}{\prod_{\ell_{2}=0}^{d_{2}-1}\left[1-e^{\left.-n^{*} q^{\ell-r_{2}-\ell_{2}}\right]}\right.} \\
\quad \vdots \quad \sum_{r_{u}=r_{u-1}+d_{u-1}+1}^{\infty} \sum_{d_{u}=1}^{\infty} \frac{\exp \left[-n^{*} q^{\ell-r_{u}-d_{u}+1}\left(1-q^{d_{u}}\right) / p\right]}{\prod_{\ell_{u}=0}^{d_{u}-1}\left[1-e^{\left.-n^{*} q^{\ell-r_{u}-\ell_{u}}\right]} .\right.}
\end{gathered}
$$

Also

$$
1-F_{n}(u) \sim p_{n}(u+1) \frac{1}{\prod_{k=1}^{\infty}\left[1-e^{-n^{*} q^{\ell-r} u+1^{-d} u+1^{-k}}\right]},
$$

The mean number of gaps $\mathbb{E}(U)$ is given by

$$
\mathbb{E}(U)=\sum_{u=0}^{\infty}\left[1-F_{n}(u)\right] .
$$

Proof: Again, we use the Poisson property. Urn $\ell$ is not empty. Above $\ell$, all urns are empty. Below $\ell$ all urns are non-empty, but we have $u$ empty gaps, starting at $\ell-r_{k}$, ending at $\ell-r_{k}-d_{k}+1$, of size $d_{k}$ each. The proof of (3) goes as follows: We have $u+1$ gaps, ending at $\ell-r_{u+1}-d_{u+1}+1$. Below, we have one non-empty urn, and below, we don't care, and cancel the corresponding part of the previous

$$
\prod_{v=1}^{\infty}\left[1-e^{-n^{*} q^{\ell-v}}\right] .
$$

$p_{n}(u)$ is a harmonic sum, that we can analyze as in (11), (13), (12), using Mellin transforms. Set $\eta:=\ell-\log n^{*}$. Then $p_{n}(u) \sim \sum_{\ell=1}^{\infty} f_{0}(\eta, u)$, with

$$
f_{0}(\eta, u)=\left[1-e^{-e^{-L \eta}}\right] e^{-\beta e^{-L \eta}} \prod_{v=1}^{\infty}\left[1-e^{-e^{-L(\eta-v)}}\right]
$$




$$
\begin{aligned}
& \sum_{r_{1}=1}^{\infty} \sum_{d_{1}=1}^{\infty} \frac{\exp \left[-e^{-L\left(\eta-r_{1}-d_{1}+1\right)}\left(1-q^{d_{1}}\right) / p\right]}{\prod_{\ell_{1}=0}^{d_{1}-1}\left[1-e^{\left.-e^{-L\left(\eta-r_{1}-\ell_{1}\right)}\right]}\right.} \\
& \sum_{r_{2}=r_{1}+d_{1}+1}^{\infty} \sum_{d_{2}=1}^{\infty} \frac{\exp \left[-e^{-L\left(\eta-r_{2}-d_{2}+1\right)}\left(1-q^{d_{2}}\right) / p\right]}{\prod_{\ell_{2}=0}^{d_{2}-1}\left[1-e^{\left.-e^{-L\left(\eta-r_{2}-\ell_{2}\right)}\right]}\right.} \\
& \vdots \sum_{r_{u}=r_{u-1}+d_{u-1}+1}^{\infty} \sum_{d_{u}=1}^{\infty} \frac{\exp \left[-e^{-L\left(\eta-r_{u}-d_{u}+1\right)}\left(1-q^{d_{u}}\right) / p\right]}{\prod_{\ell_{u}=0}^{d_{u}-1}\left[1-e^{\left.-e^{-L\left(\eta-r_{u}-\ell_{u}\right)}\right]}\right.} .
\end{aligned}
$$

Set

$$
\begin{aligned}
\phi(\alpha, u) & :=\int_{-\infty}^{\infty} e^{\alpha \eta} f_{0}(\eta, u) d \eta \\
\Upsilon_{0}^{*}(s, u) & =\left.L \phi(\alpha, u)\right|_{\alpha=-L s}, \\
w_{0}(u) & =\frac{1}{L} \sum_{\ell \neq 0} \Upsilon_{0}^{*}\left(\chi_{\ell}, u\right) e^{-2 \ell \pi \mathbf{i} \log n^{*}} .
\end{aligned}
$$

Then we obtain the periodic contribution:

Theorem 6.3 $p_{n}(u) \sim \phi(0, u)+w_{0}(u)+\mathcal{O}\left(n^{-C}\right), C>0 ; w_{0}(u)$ is a periodic small function of $\log n^{*}$.

Similarly, starting from (3), we set

$$
\begin{aligned}
f_{M}(\eta) & :=\sum_{u=0}^{\infty} \frac{f_{0}(\eta, u+1)}{\prod_{k=1}^{\infty}\left[1-e^{\left.-e^{-L\left(\eta-r_{u+1}-d_{u+1}-k\right)}\right]}\right.} \\
\phi_{M}(\alpha) & :=\int_{-\infty}^{\infty} e^{\alpha \eta} f_{M}(\eta) d \eta \\
\Upsilon_{0, M}^{*}(s) & =\left.L \phi_{M}(\alpha)\right|_{\alpha=-L s}, \\
w_{0, M} & =\frac{1}{L} \sum_{\ell \neq 0} \Upsilon_{0, M}^{*}\left(\chi_{\ell}\right) e^{-2 \ell \pi \mathbf{i} \log n^{*}} .
\end{aligned}
$$

Then

\section{Theorem 6.4}

$$
\mathbb{E}(U) \sim \phi_{M}(0)+w_{0, M}+\mathcal{O}\left(n^{-C_{M}}\right), \quad C_{M}>0
$$

Also, we can consider the case where all gaps do have a length $\leq m$. Set $M L:=$ maximum gaps length and $F_{g}(m):=\mathbb{P}[M L \leq m]$. This leads to

$$
\begin{gathered}
F_{g}(m) \sim \sum_{u=0}^{\infty} \sum_{\ell=1}^{\infty}\left[1-e^{-n^{*} q^{\ell}}\right] e^{-n^{*} q^{\ell+1} / p} \prod_{v=1}^{\infty}\left[1-e^{-n^{*} q^{\ell-v}}\right] \\
\sum_{r_{1}=1}^{\infty} \sum_{d_{1}=1}^{m} \frac{\exp \left[-n^{*} q^{\ell-r_{1}-d_{1}+1}\left(1-q^{d_{1}}\right) / p\right]}{\prod_{\ell_{1}=0}^{d_{1}-1}\left[1-e^{-n^{*} q^{\ell-r_{1}-\ell_{1}}}\right]}
\end{gathered}
$$




$$
\begin{aligned}
& \quad \sum_{r_{2}=r_{1}+d_{1}+1}^{\infty} \sum_{d_{2}=1}^{m} \frac{\exp \left[-n^{*} q^{\ell-r_{2}-d_{2}+1}\left(1-q^{d_{2}}\right) / p\right]}{\prod_{\ell_{2}=0}^{d_{2}-1}\left[1-e^{-n^{*} q^{\ell-r_{2}-\ell_{2}}}\right]} \\
& \vdots \\
& \quad \sum_{r_{u}=r_{u-1}+d_{u-1}+1}^{\infty} \sum_{d_{u}=1}^{m} \frac{\exp \left[-n^{*} q^{\ell-r_{u}-d_{u}+1}\left(1-q^{d_{u}}\right) / p\right]}{\prod_{\ell_{u}=0}^{d_{u}-1}\left[1-e^{-n^{*} q^{\ell-r_{u}-\ell_{u}}}\right]},
\end{aligned}
$$

and setting

$$
\begin{aligned}
& f_{1}(\eta, m)=\sum_{u=0}^{\infty}\left[1-e^{-e^{-L \eta}}\right] e^{-\beta e^{-L \eta}} \prod_{v=1}^{\infty}\left[1-e^{-e^{-L(\eta-v)}}\right] \\
& \sum_{r_{1}=1}^{\infty} \sum_{d_{1}=1}^{m} \frac{\exp \left[-e^{-L\left(\eta-r_{1}-d_{1}+1\right)}\left(1-q^{d_{1}}\right) / p\right]}{\prod_{\ell_{1}=0}^{d_{1}-1}\left[1-e^{-e^{-L\left(\eta-r_{1}-\ell_{1}\right)}}\right]} \\
& \sum_{r_{2}=r_{1}+d_{1}+1}^{\infty} \sum_{d_{2}=1}^{m} \frac{\exp \left[-e^{-L\left(\eta-r_{2}-d_{2}+1\right)}\left(1-q^{d_{2}}\right) / p\right]}{\prod_{\ell_{2}=0}^{d_{2}-1}\left[1-e^{-e^{-L\left(\eta-r_{2}-\ell_{2}\right)}}\right]} \\
& \vdots \\
& \sum_{r_{u}=r_{u-1}+d_{u-1}+1}^{\infty} \sum_{d_{u}=1}^{m} \frac{\exp \left[-e^{-L\left(\eta-r_{u}-d_{u}+1\right)}\left(1-q^{d_{u}}\right) / p\right]}{\prod_{\ell_{u}=0}^{d_{u}-1}\left[1-e^{-e^{-L\left(\eta-r_{u}-\ell_{u}\right)}}\right]}, \\
& \phi(\alpha, m):=\int_{-\infty}^{\infty} e^{\alpha \eta} f_{1}(\eta, m) d \eta, \\
& \Upsilon_{0}^{*}(s, m)=\left.L \phi(\alpha, m)\right|_{\alpha=-L s}, \\
& w_{0}(m)=\frac{1}{L} \sum_{\ell \neq 0} \Upsilon_{0}^{*}\left(\chi_{\ell}, m\right) e^{-2 \ell \pi \mathbf{i} \log n^{*}} .
\end{aligned}
$$

We have the periodic contibution:

\section{Theorem 6.5}

Also

$$
\begin{gathered}
F_{g}(m) \sim \phi(0, m)+w_{0}(m)+\mathcal{O}\left(n^{-C_{1}}\right), \quad C_{1}>0 . \\
\mathbb{E}(M L)=\sum_{m=0}^{\infty}\left[1-F_{g}(m)\right] .
\end{gathered}
$$

Let us now return to our joint distribution of $X_{T}$ and $S_{T}$ (independently of $T$ ). Set

$$
p_{n}(i, j, k):=\mathbb{P}\left[X_{T}=i, S_{T}=j\right] .
$$

Setting again $\eta=\ell-\log n^{*}$, this leads to

$$
\begin{aligned}
f_{2}(\eta, i, j, k) & =e^{-e^{-L \eta}} \frac{\left(e^{-L \eta}\right)^{i}}{i !} e^{-\beta e^{-L \eta}\left(1-q^{k}\right)} e^{-\beta e^{-L(\eta+k)}} \frac{\left(\beta e^{-(\eta+k)}\right)^{j}}{j !} \times \\
& \sum_{u=0}^{\infty} \prod_{v=1}^{\infty}\left[1-e^{-e^{-L(\eta-v)}}\right]
\end{aligned}
$$




$$
\begin{gathered}
\sum_{r_{1}=1}^{\infty} \sum_{d_{1}=1}^{k-1} \frac{\exp \left[-e^{-L\left(\eta-r_{1}-d_{1}+1\right)}\left(1-q^{d_{1}}\right) / p\right]}{\prod_{\ell_{1}=0}^{d_{1}-1}\left[1-e^{\left.-e^{-L\left(\eta-r_{1}-\ell_{1}\right)}\right]}\right.} \\
\sum_{r_{2}=r_{1}+d_{1}+1}^{\infty} \sum_{d_{2}=1}^{k-1} \frac{\exp \left[-e^{-L\left(\eta-r_{2}-d_{2}+1\right)}\left(1-q^{d_{2}}\right) / p\right]}{\prod_{\ell_{2}=0}^{d_{2}-1}\left[1-e^{\left.-e^{-L\left(\eta-r_{2}-\ell_{2}\right)}\right]}\right.} \\
\vdots \\
\sum_{r_{u}=r_{u-1}+d_{u-1}+1}^{\infty} \sum_{d_{u}=1}^{k-1} \frac{\exp \left[-e^{-L\left(\eta-r_{u}-d_{u}+1\right)}\left(1-q^{d_{u}}\right) / p\right]}{\prod_{\ell_{u}=0}^{d_{u}-1}\left[1-e^{\left.-e^{-L\left(\eta-r_{u}-\ell_{u}\right)}\right]},\right.} \\
\Upsilon_{0}^{*}(s, i, j, k)= \\
w_{0}(i, j, k)= \\
\int_{-\infty}^{\infty} e^{\alpha \eta} f_{2}(\eta, i, j, k) d \eta, \\
L
\end{gathered}
$$

We have the asymptotics:

Theorem 6.6

$$
p_{n}(i, j, k) \sim \phi(0, i, j, k)+w_{0}(i, j, k)+\mathcal{O}\left(n^{-C_{2}}\right), \quad C_{2}>0 .
$$

Of course, most of our expressions are rather complicated, but they are explicit, with their periodic contribution and it doesn't seem possible to simplify them further on.

\section{Conclusion}

Taking $k \rightarrow \infty$ in our asymptotic results, we find that $\mathbb{E}_{n}\left(X_{T}\right) \sim \frac{p}{q L}, \mathbb{E}_{n}\left(S_{T}\right) \sim 0$, and $\mathbb{E}_{n}(T) \sim$ $\log n+\frac{1}{2}+\frac{\gamma}{L}$. (All quantities are given without the tiny fluctuations.) These values are intuitive, as the first one corresponds to the average number of (tied) winners (see (9)), the second one is clearly 0 , and the third one is the average value of the maximum of $n$ geometrically distributed random variables, which is a very well studied quantity.

Our asymptotic results contain the numbers of interest on the righthand side. This looks paradoxical at first glance, but it is very common in combinatorial enumeration. The series involved converge very quickly, and one only has to compute a few values for $a_{n}$ from the recursion to obtain some reasonable accuracy.

Using some results from our previous papers, we also get asymptotic joint distributions related to $T, X_{T}, S_{T}$. This also provides some asymptotics on gaps properties. It remains to solve recurrence (2) and to simplify some expressions.

\section{Acknowledgement}

The pertinent comments of two referees led to improvements in the presentation. 


\section{References}

[1] L. Bondesson, T. Nilsson, and G. Wikstrand. Probability calculus for silent elimination; a method for medium access control. to appear, 2007.

[2] T. Bruss and C. A. O'Cinneide. On the maximum and its uniqueness for geometric random samples. J. Appl. Probab., 27:598-610, 1990.

[3] B. Eisenberg, G. Stengle, and G. Strang. The asymptotic probability of a tie for first place. Ann. Appl. Probab., 3:731-745, 1993.

[4] P. Flajolet and G. N. Martin. Probabilistic counting algorithms for data base applications. Journal of Computer and System Sciences, 31:182-209, 1985.

[5] W.M.Y. Goh and P. Hitczenko. Gaps in samples of geometric random variables. Discrete Mathematics, 307:2871-2890, 2007.

[6] P. Hitczenko and A. Knopfmacher. Gap-free samples of geometric random variables. Discrete Mathematics, 294:225-239, 2005.

[7] P. Hitczenko and G. Louchard. Distinctness of compositions of an integer: a probabilistic analysis. Random Structures and Algorithms, 19(3,4):407-437, 2001.

[8] P. Kirschenhofer and H. Prodinger. A result in order statistics related to probabilistic counting. Computing, 51:15-27, 1993.

[9] P. Kirschenhofer and H. Prodinger. The number of winners in a discrete geometrically distributed sample. Annals in Applied Probability, 6:687-694, 1996.

[10] M. Loève. Probability Theory. D. Van Nostrand, 1963.

[11] G. Louchard and H. Prodinger. Asymptotics of the moments of extreme-value related distribution functions. Algorithmica, 46:431-467, 2006. Long version: http://www.ulb.ac.be/di/mcs/louchard/moml.ps.

[12] G. Louchard and H. Prodinger. On gaps and unoccupied urns in sequences of geometrically distributed random variables. Discrete Mathematics, 308,9:1538-1562, 2008. Long version: http://www.ulb.ac.be/di/mcs/louchard/gaps18.ps.

[13] G. Louchard, H. Prodinger, and M.D. Ward. The number of distinct values of some multiplicity in sequences of geometrically distributed random variables. Discrete Mathematics and Theoretical Computer Science, AD:231-256, 2005. 2005 International Conference on Analysis of Algorithms.

[14] W. Szpankowski. Average Case Analysis of Algorithms on Sequences. Wiley, New York, 2001.

[15] W. Szpankowski and V. Rego. Yet another application of a binomial recurrence. Order statistics. Computing, 43(4):401-410, 1990. 